\title{
The role of focus marking in disjunctive questions: A QUD-based approach*
}

\author{
Morwenna Hoeks \\ University of California Santa Cruz.
}

\begin{abstract}
Disjunctive questions are ambiguous: they can either be interpreted as polar questions (PolQs), as open disjunctive questions (OpenQs), or as closed alternative questions (ClosedQ). The goal of this paper is to show that the difference in interpretation between these questions can be derived via effects of focus marking directly. In doing so, the proposal brings out the striking parallel between the prosody of questions with foci/contrastive topics on the one hand and that of alternative questions on the other. Unlike previous approaches, this proposal does not rely on structural differences between AltQs and PolQs derived via ellipsis or syntactic movement. To show how this works out, an account of focus and contrastive topic marking in questions is put forward in which f-marking in questions determines what constitutes a possible answer by signaling what the speaker's QUD is like. By imposing a congruence condition between f-marked questions and their answers that requires answers to resolve the question itself as well as its signaled QUD, we predict the right answerhood conditions for disjunctive questions.
\end{abstract}

Keywords: focus, disjunction, alternative semantics, questions, contrastive topics, intonation

\section{Introduction}

Disjunctive questions such as (1) are well-known to be ambiguous (Bartels 1999; Han \& Romero 2004; Beck 2006; Pruitt \& Roelofsen 2013; Roelofsen 2015; Meertens 2019).

Did Tony drink coffee or tea?

$\checkmark$ PolQ, $\checkmark$ ClosedQ $\checkmark$ OpenQ

Questions like (1) can be disambiguated by prosody. For example, when (1) is pronounced with a rising boundary tone and a flat intonation on the disjunction, it is interpreted as a polar question (PolQ), which allows for both "yes" and "no" responses as shown in (2) below.
a. A: Did Tony drink coffee-or-tea yesterday morning? ${ }^{\mathrm{H}-\mathrm{H} \%}$
$\checkmark$ PolQ
b. B: Yes, he did.
c. B: No, he didn't.

This PolQ reading disappears when each disjunct is pitch accented and there is a phrasal break before the final disjunct. For example, in (3) the first disjunct receives a rising pitch accent

* I am grateful to Maziar Toosarvandani, Dean McHugh, Hana Kalpak, Jonathan Pesetsky, Donka Farkas, Adrian Brasoveanu and Floris Roelofsen for their feedback. I would also like to thank audiences at SALT30, ESSLLI 2019 and the InqBnB3 workshop, in particular Scott AnderBois, Beste Kamali and Satoshi Tomioka for valuable discussion. All errors are mine. 
and the second disjunct receives a falling pitch accent, followed by a falling boundary tone. Throughout, I will use small caps to indicate the presence of pitch accents.
a. A: Did Tony drink COFFEE ${ }^{\mathrm{H}^{*}}$ or $\mathrm{TEA}^{\mathrm{H}+\mathrm{L} *}$ yesterday morning? ${ }^{\mathrm{L}-\mathrm{L} \%} \checkmark \checkmark$ ClosedQ
b. \#B: Yes/No
c. B: He drank COFFEE/TEA
d. \#B: He drank WATER

In this case the PolQ reading is out: A simple yes/no response is infelicitous. ${ }^{1}$ Intuitively, this is because such answers do not seem to provide enough information to fully resolve the question that is being asked. Instead, answers that correspond to each of the disjuncts like (3c), form a natural response to these questions. Answers that do not entail any of the disjuncts, like (3d), do not. I will call this type of question a closed alternative question (ClosedQ).

ClosedQs can be distinguished from what I will call open alternative questions (OpenQs): disjunctive questions with a rising pitch accent on each disjunct followed by a rising boundary tone, as shown in (4).
a. A: Did Tony drink COFFEE ${ }^{(\mathrm{L}+) \mathrm{H}^{*}}$ or TEA ${ }^{\left((\mathrm{L}+) \mathrm{H}^{*}\right.}$ yesterday morning $?^{\mathrm{H}-\mathrm{H} \%} \checkmark$ OpenQ
b. \#B: Yes
c. B: No
d. B: He drank COFFEE/TEA
e. B: He drank WATER

OpenQs are like ClosedQs in that they disallow simple affirmative responses as in (4b) and allow for more informative responses that correspond to each disjunct as in (4d). However, OpenQs are like PolQs in that they allow for negative responses as in (4c), as well as more informative negative responses such as those in (4e).

In short, the answerhood conditions of disjunctive questions depend on the way in which they are pronounced. In a Hamblin-style semantics, the difference in interpretation between a disjunctive PolQ, an OpenQ and a ClosedQ is roughly the following (where I use $|\varphi|$ to abbreviate the set of worlds in which the sentence $\varphi$ is true).

$$
\begin{array}{ll}
\text { a. } & \llbracket(2) \rrbracket=\{\mid \text { T drinks tea or coffee }|,| \text { T drinks neither } \mid\} \\
\text { b. } & \llbracket(3) \rrbracket=\{\mid \text { T drinks (only) tea }|,| \text { T drinks (only) coffee } \mid\} \\
\text { c. } & \llbracket(4) \rrbracket=\{\mid \text { T drinks (only) tea }|,| \text { T drinks (only) coffee }|,| \text { T drinks neither } \mid\}
\end{array}
$$

From this, we can note that both in terms of their prosody and in terms of their interpretation, disjunctive questions vary along two dimensions. First, we see that the disjunction provides alternatives in the form of distinct answers in open and closed alternative questions but it does not do so in PolQs. Moreover, it seems that ClosedQs and OpenQs (but not PolQs) come with an exclusivity inference on the disjunction suggesting that only one of the two disjuncts is true. Prosodically, these distinctions are expressed by the presence or absence of pitch accents: in both types of alternative questions the disjuncts are pitch accented, but the disjuncts in a

1 In this paper, I am not concerned with the use of polarity particles per se. Instead, the goal of this paper is simply to account for patterns in the semantic content of possible answers. 
PolQ are crucially not. Because OpenQs and ClosedQs can be grouped together in this way, I will refer to them both as alternative questions (AltQs) from here on.

Second, we see that PolQs and OpenQs also pattern together, because both their denotations contain answers that do not entail the disjunction, while ClosedQs do not allow for such an answers. This distinction is again expressed prosodically, because both PolQs and OpenQs come with a rising boundary tone and pitch accents, while ClosedQs always have a falling boundary tone and final pitch accent.

Since their prosody is the only difference in the form of these questions, explaining these differences in interpretation will have to involve an explicit account of the role of prosody in determining the meaning of disjunction, and questions more generally.

In this paper, I will do this by making the crucial assumption that focus marking is the driving force behind these differences. That is, I will assume that the pitch accents we find in AltQs are similar to those in a wh-question (WhQ) like (6a), or a PolQ like (6b), which are typically assumed to involve focus or contrastive topic marking (Constant 2014; Kamali \& Krifka 2020).
a. Who did SOPHIE ${ }^{\mathrm{L}+\mathrm{H}^{*}}$ invite to the party?
b. Did SOPHIE ${ }^{\mathrm{L}+\mathrm{H}^{*}}$ invite Graham to the party?

Although the phenomenon of focus marking has often been studied in assertions, focus marking inside questions has received less attention (but see Kamali \& Krifka (2020) for a recent proposal). I will therefore first put forward a general account of focus marking in questions, which crucially relies on the idea that f-marking in questions affects the implicit "question under discussion" (QUD). That is, just like foci in assertions, foci in questions signal what the QUD looks like. The shape of this QUD will then affect what is considered a proper answer to the question that triggers it. In a nutshell, answers to f-marked questions have to resolve the question itself, but should also address the implicit QUD that the question is signalled to be part of. In this way, implicit QUDs will have a direct effect on the answerhood conditions of a question.

The main claim of this paper will therefore be that by placing disjunctive questions in a broader context of focus marking, we can understand how prosody affects the meaning of these questions in a more systematic way. Moreover, previous approaches have assumed that syntactic differences between PolQs and AltQs give rise to their differences in interpretation, but the current proposal does not rely on any structural differences between disjunctive questions (see Hoeks (2019) for some problems with syntactic approaches). Although accounts of some of these contrasts involving disjunctive questions have already been proposed before (Roelofsen \& van Gool 2009; Biezma 2009; Han \& Romero 2004; Beck 2006), to my knowledge, none of these proposals takes into account the whole paradigm in a way that brings out the striking parallel between the prosody of disjunctive questions on the one hand, and that of questions with focus and/or contrastive topic marking on the other. An additional advantage of accounting for the meaning of AltQs by relying on focus marking is that it also allows us to account for additional inferences that come with AltQs, such as the exhaustivity inference of ClosedQs, and the lack thereof in OpenQs/PolQs.

The remainder of this paper is structured as follows. To illustrate how we can think of the 
effect of focus marking in questions, in Section 2 I will first discuss the effect of contrastive topic marking which has already been explicitly studied in both questions and assertions (Büring 2003; Büring 2016; Constant 2014; Wagner 2012). I will use contrastive topics to put forward an account of focus marking that is generalized to questions. In Section 3 we will then apply this account to simple PolQs, and in Section 4 we will see how we can apply such an account to capture the effect of focus in disjunctive questions too. Finally, in Section 5 I will conclude.

\section{Background on contrastive topics}

In this section I will use contrastive topics to introduce some crucial assumptions about focus marking in general. Throughout, I will adopt a standard Roothian account of focus marking in which we keep track of focus alternatives by assuming that a focused expression has both an ordinary semantic value and a focus semantic value (Rooth 1985, 1992). Very much in line with original the proposal by Rooth, I will also assume that the focus alternatives generated in the f-value of an expression are interpreted using a squiggle operator (denoted by $\sim$ ) which establishes an anaphoric relation between the focus alternatives and a focus antecedent. Since I will only be dealing with focus antecedents that are questions here, I will refer to this focus antecedent as the QUD. We can thus write Rooth's original squiggle operator as follows:

(7) Squiggle (Rooth 1992)

a. $\quad \llbracket \sim \varphi \rrbracket^{o}=\llbracket \varphi \rrbracket^{o}$

b. $\quad \llbracket \sim \varphi \rrbracket^{f}=\left\{\llbracket \varphi \rrbracket^{o}\right\}$

c. and presupposes that the context contains a $Q U D$ such that

1. $Q U D \subseteq \llbracket \varphi \rrbracket^{f}$

2. $|Q U D|>2$

3. $\llbracket \varphi \rrbracket^{o} \in Q U D$

In other words, the squiggle operator is responsible for the ensuring that the set of focus alternatives triggered by its prejacent is a subset of the QUD, and that the ordinary value of its prejacent provides an answer to that same QUD.

In focus marked expressions that form answers to questions, the focus antecedent is provided by the preceding question. For instance, the explicitly raised WhQ in (8) is a subset of the focus alternatives of the answer.

a. A: Who did Sophie invite to the party?

b. B Sophie invited GRAHAM .

$\rightsquigarrow Q U D \subseteq$ Who did Sophie invite to the party?

Just like focus marking, requirements on contrastive topic marking in answers can also be explained in terms of coherence requirements between question/answer pairs. To see this, consider first the answer in (9b) which constitutes a typical use of a contrastive topic.

(9) a. A: Who invited whom to the party? 
b. B: TONY $\mathrm{CT}^{\mathrm{L}+\mathrm{H}^{*}}$ invited SOPHIE $\mathrm{H}^{\mathrm{H}}$ and $\mathrm{TOM}_{\mathrm{CT}}^{\mathrm{L}+\mathrm{H}^{*}}$ invited ANNEY $\mathrm{F}^{\mathrm{H}} \mathrm{L}-\mathrm{L} \%$

Contrastive topics like Tony and Tom are generally marked with an $\mathrm{L}+\mathrm{H}^{*}$ pitch accent and typically co-occur with regular foci that are marked with $\mathrm{H}^{*}$ accents. I will refer to such sentences as "CT-F" sentences.

The implementation of the effect of contrastive topics differs from one theory to the next. Here I will follow Büring (2003) and Constant (2014) in assuming that the contrastive topic alternatives give rise to nested sets of alternatives, i.e sets of sets of propositions. The intuition behind this is that contrastive topics signal a certain strategy of answering an overarching question. Such a strategy itself consists of a series of questions that one could ask to resolve the overarching question itself. Thus, instead of presupposing a QUD that is a question-as would be the case with simple F-marked assertions- these CT-F sentences presuppose the presence of what I will refer to as a superquestion, i.e. a set of questions. In the case of (9b), the speaker signals that they are answering the preceding double WhQ by answering a series of WhQs of the form Who did $x$ invite to the party? In other words, the f-value of (9b) will correspond to the set of questions in (10).

$$
\left.\llbracket \text { TONY }_{C T} \text { invited SOPHIE } F \rrbracket^{f}=\{\mid x \text { invited } y \mid: y \in \text { PEOPLE }\}: x \in \text { PEOPLE }\right\}
$$

In Büring (2003), it is assumed that an assertion with contrastive topic and focus marking has to be congruent to such a superquestion, that is, it has to answer a subquestion within it. Because of these parallels between focus and contrastive topic marking, Constant (2014) generalizes the entry for $\sim$ to capture its effect for contrastive topics too:

\section{Generalized squiggle (Constant 2014):}

$$
\begin{array}{ll}
\text { a. } & \llbracket \sim \varphi \rrbracket^{o}=\llbracket \varphi \rrbracket^{o} \\
\text { b. } & \llbracket \sim \varphi \rrbracket^{f}=\left\{\llbracket \varphi \rrbracket^{o}\right\} \\
\text { c. } & \text { and presupposes that the context contains a } Q U D \text { such that }
\end{array}
$$

1. $Q U D \subseteq \llbracket \varphi \rrbracket^{f}$

2. $|Q U D|>2$

3. $\llbracket \varphi \rrbracket^{o} \in^{*} Q U D$

where $\epsilon^{*}$ means: "contains somewhere within it"

Note that the only difference between Rooth's definition of the squiggle and that of Constant is the very last clause, which now states that $\llbracket \varphi \rrbracket^{o}$ has to either answer the QUD (if the QUD is a question), or answer a subquestion within that QUD (if that QUD is a set of questions). For instance, the first conjunct in the answer in (9b) answers the subquestion Who did Tony invite?, while the second conjunct answers Who did Tom invite?

Although not mentioned by Büring (2003) or Wagner (2012), Constant (2014) observes that contrastive topics can also occur in questions. Consider for instance the WhQ in (12a) below with contrastive topic marking on Tony.

a. A: Who did TONY $_{\mathrm{CT}}$ invite to the party?

b. B: Tony ${ }_{\mathrm{CT}}$ invited Sophie ${ }_{\mathrm{F}}$

In this case, CT-marking is also assumed to give rise to a set of f-alternatives which are 
questions of the form Who did $x$ invite to the party. In other words, the question in (12a) raises the WhQ in (13a), and at the same time, presupposes a QUD which corresponds to the superquestion in (13b).

$$
\begin{array}{ll}
\text { a. } & {[[(12 \mathrm{a})]]^{o}=\{\mid \text { Tony invited } x \mid: x \in \text { HUMAN }\}} \\
\text { b. } & {[[(12 \mathrm{a})]]^{f}=\{\{\mid \mathrm{y} \text { invited } x \mid: y \in \text { HUMAN }\}: x \in \text { HUMAN }\}}
\end{array}
$$

The answer in (12b) gives rise to the exact same QUD as the question itself. Therefore, the answer in (12b) answers $\left[[(12 \mathrm{a})]^{o}\right.$, which also happens to be a question within both the superquestions $[[(12 \mathrm{a})]]^{f}$ and $[[(12 \mathrm{~b})]]^{f}$.

This latter fact, I argue, is not a coincidence. Note that the question/answer pair above becomes infelicitous when the answer presupposes a different QUD as the one signalled by the question. This is illustrated in (14).
a. A: Who did TONY $C T$ invite to the party?
b. B: \#SOPHIE $C T$ was invited by TONY $_{F}$

Although the answer in (14b), answers the preceding question itself, it now gives rise to a superquestion that is different than the one presupposed by the question. That is, the answer presupposes as its QUD a set of WhQs of the form Who was x invited by?:

$$
\left.\llbracket \text { SOPHIE }_{C T} \text { was invited by TONY } \rrbracket^{f}=\{\mid y \text { invited } x \mid: y \in \text { PEOPLE }\}: x \in \text { PEOPLE }\right\}
$$

The infelicity of examples like this suggests that whenever a question already presupposes a particular QUD via CT-marking, its answer has to be congruent to that same QUD.

Note that when we just look at answers to questions which are not themselves CT-marked, we do not see this pattern: the same answer to the double WhQ we saw in (9a) could have presupposed a different QUD, as shown in (16).
a. A: Who invited whom to the party?
b. B: Sophie $C T$ was invited by Tony $F$ and Anney ${ }_{C T}$ was invited by Tom $_{F}$

This is because the double WhQ, unlike a CT-marked question, is compatible with both the superquestions in (10) or (15). By asking the double WhQ itself, the speaker wants to know who invited whom and thus wants to see the question in (17) resolved, but this question itself does not specify in which way this question should be addressed (Büring 2003). That is, one could answer (17) either by answering all the WhQs questions in (10) or those in (15).

$$
[[\text { Who invited whom }]]=\{\mid x \text { invited } y \mid: x, y \in \text { HUMAN }\}
$$

In short, for a CT-marked question/answer pair to be coherent, we need the answer to presuppose the same QUD as is already presupposed by the question.

I suggest that the intuition behind this is as follows: if a speaker signals their question to be part of a specific overarching QUD, a fully cooperative addressee will view the explicitly raised question as being part of that same QUD. In answering the speaker's question, such an addressee would then not only provide an answer that is informative enough to resolve the 
speaker's question, but will also address the correct QUD.

To capture this intuition, I will take a slightly different approach to contrastive topic congruence, as I will capture its requirements as being part of general conditions on what constitutes a proper answer. I then adopt a generalized $\sim$ too, but instead of capturing the fact that $\llbracket \varphi \rrbracket^{o} \in Q U D$ as an effect of the squiggle, I will analyze this as a general requirement for answers to be informative enough to resolve the raised QUD. The new version of $\sim$ will only be responsible for matching the f-alternatives with a focus antecedent, but will not have anything to say about the relationship between an expression's o-value and f-value: ${ }^{2}$

\section{Generalized squiggle:}
a. $\quad \llbracket \sim \varphi \rrbracket^{o}=\llbracket \varphi \rrbracket^{o}$
b. $\quad \llbracket \sim \varphi \rrbracket^{f}=\llbracket \varphi \rrbracket^{o}$
c. $\quad$ and presupposes that the context contains a $Q U D$ such that $Q U D \subseteq \llbracket \varphi \rrbracket^{f}$

In addition, I then define proper answerhood in the following way:

Focus-sensitive answerhood: An answer A properly answers a question $Q$ iff

a. $\llbracket A \rrbracket^{o}$ resolves $\llbracket Q \rrbracket^{o}$,

b. $\llbracket A \rrbracket^{f} \subseteq Q U D_{Q}$, and $\quad$ where $Q U D_{Q}$ is the salient $Q U D$ signalled by $Q$

c. $\llbracket A \rrbracket^{o}$ resolves (one subquestion within) $\llbracket A \rrbracket^{f}$.

I will adopt an inquisitive notion of answerhood, thus taking into account that answers which entail any of the question-alternatives will also answer the question itself (Ciardelli, Groenendijk \& Roelofsen 2018).

(20) Resolution: A proposition A resolves a set of propositions Q iff A $\subseteq$ A' for some A' $\in \mathrm{Q}$.

Let us see how this works out for the CT-marked WhQ and its answer, repeated here in (21).
a. A: Who did $\mathrm{TONY}_{\mathrm{CT}}$ invite to the party?
b. B: Tony ${ }_{\mathrm{CT}}$ invited Sophie $\mathrm{F}_{\mathrm{F}}$

By the definition of $\sim$, the question first establishes that the salient $Q U D \subseteq \llbracket Q \rrbracket^{f}$. Then due to focus-sensitive answerhood, $\llbracket A \rrbracket^{f} \subseteq Q U D_{Q}$, meaning that $\llbracket A \rrbracket^{f} \subseteq \llbracket Q \rrbracket^{f}$. The denotation of the answer itself, i.e. $\llbracket A \rrbracket^{o}$ then has to resolve the question itself and address $Q U D_{Q}$, in this case by addressing at least one subquestion within it. Note that, since in this case $\llbracket Q \rrbracket^{o} \in \llbracket Q \rrbracket^{f}$, this will always be the case for any answer that resolves $\llbracket Q \rrbracket^{o}$.

Using focus-sensitive answerhood, we can capture our familiar question/answer congruence too if we assume that in the absence of f-marking in the question the contextual QUD is given by the question itself.

In conclusion, an answer to a CT-marked question should be congruent to the f-value of

2 Note that, instead of resetting the f-value of the singleton set containing the o-value of its prejacent, this version of the squiggle only resets the f-value to its o-value. This is because I will treat the f-value of non f-marked expressions not as singleton sets, but simply as being the same as their o-value. See Section 3 for details. 
that question; an answer to a non f-marked question should be congruent to that question itself.

We have now seen examples of contrastive topics in questions and answers, as well as foci in answers. Below, I will argue that foci occur in questions as well, and can be analyzed in the same way as we think of contrastive topics in questions. I will do this first by considering simple PolQs, which I will argue can receive both contrastive topic and focus marking. Having an explicit account of focus and contrastive topic marking in place for PolQs will then help us understand how the meaning of disjunctive questions is affected by the presence of these foci as well.

\section{Focus and contrastive topic marking in PolQs}

Although contrastive topic marking has been discussed for WhQs as the ones we saw above, we also find prominences in simple PolQs like (22a).

$$
\begin{aligned}
& \text { a. A: Did SOPHIE } \mathrm{CT}_{\mathrm{CT}}^{\mathrm{L}+\mathrm{H}^{*}} \text { go to the party }{ }^{\mathrm{H} \%} \\
& \text { b. B: } \operatorname{SOPHIE}_{\mathrm{CT}}^{\mathrm{L}+\mathrm{H}^{*}} \operatorname{DID}_{\mathrm{F}}^{\mathrm{H}^{*}} \text {, but TONY } \mathrm{CT}^{\mathrm{L}+\mathrm{H}^{*}} \operatorname{DIDN}^{\prime} \mathrm{T}_{\mathrm{F}}^{\mathrm{H}^{*} \mathrm{~L}-\mathrm{L} \%}
\end{aligned}
$$

In this section I will show how we can derive the effects of these questions in the same way as contrastive topics in WhQs. That is, just like the CT-marked WhQ in (12a) gave rise to a QUD which was a set of WhQs, we can think of the effect of (22a) as giving rise to a set of PolQs. I will thus assume that the o-value of (22a) will be the PolQ in (23a), and its f-value will be the set of alternatives in (23b):

a. $\quad \llbracket(22 \mathrm{a}) \rrbracket^{o}=\{\mid$ Sophie went to the party $|,| \neg$ Sophie went to the party $\mid\}$

b. $\llbracket(22 \mathrm{a}) \rrbracket^{f}=\{\{\mid x$ went to the party $|,| \neg x$ went to the party $\mid\}: x \in$ HUMAN $\}$

In other words, by asking (22a), the speaker not only raises the PolQ itself, but also signals this PolQ to be part of the higher-level superquestion in (23b). To derive this as our f-value, I will first develop a way to generate f-alternatives for contrastive topics in WhQs. We can then apply the same mechanism to contrastive topic marked PolQs - and later to AltQs as well.

\subsection{Deriving the CT-alternatives}

In deriving the appropriate f-values for expressions with contrastive topics, I will follow Constant (2014) and Wagner (2012) in assuming a compositional account of contrastive topics in which they are analyzed as foci that scope over another focus. The generalization proposed in Wagner (2012) is then that contrastive topics have to outscope the other focus. Concretely, a sentence like (24b) is thus analyzed as involving two foci, which will each generate their own alternatives.

$$
\begin{aligned}
& \text { a. Who did TONY } \mathrm{CT}_{\mathrm{CT}}^{\mathrm{L}+\mathrm{H}^{*}} \text { invite? } \\
& \text { b. TONY } \mathrm{Y}_{\mathrm{CT}}^{\mathrm{L}+\mathrm{H}^{*}} \text { invited SOPHIE } \mathrm{H}^{\mathrm{H} *} \mathrm{~L}-\mathrm{L} \%
\end{aligned}
$$

For this reason, I refer to both regular foci and contrastive topics as being "f-marked". For ease of presentation however, I will still distinguish between them by calling the f-marked 
constituent that takes wider scope a contrastive topic (CT), and the constituent that takes narrow scope a focus $(\mathrm{F})$, as schematized in (25a) below. I will refer to expressions that occur within the scope of a squiggle operator "on their own" as in (25b) as being F-marked as well.

$$
\begin{array}{ll}
\text { a. } & \sim[\ldots \mathrm{CT} \ldots[\ldots \mathrm{F} \ldots]] \\
\text { b. } & \sim[\ldots \mathrm{F} \ldots]
\end{array}
$$

Thus, I will refer to expressions as contrastive topics only in case these expressions take scope over another focus in the scope of a single squiggle operator. All other cases will be referred to as foci. The reason for this is that it will help us explain the mapping between prosody and these different types of foci. In Jackendoff (1972) and Büring (2003), CTs in English are characterized as constituents marked by B-accents, which are usually transcribed with $\mathrm{L}+\mathrm{H}^{*}$ pitch accents in a ToBI system. CTs are also followed by a $\mathrm{H} \%$ boundary tone. In contrast, foci are typically marked with $\mathrm{H}^{*}$ pitch accents, followed by a L-L\% boundary if the F-marked constituent is the last accented constituent in a declarative sentence (Wagner 2012). Because we can think of the L-L\% boundary tone as the default in declarative sentences, I will simply assume the following focus-prosody mapping:

\section{Focus-prosody mapping}
a. $\mathrm{CT} \rightarrow \mathrm{L}+\mathrm{H}^{*} \mathrm{H} \%$
b. $\mathrm{F} \rightarrow \mathrm{H}^{*}$

Concretely then, the CT-F answer we already saw in (24b) will have the following LF, and we correctly predict its prosody already indicated using superscripts in (24b).

$$
\sim\left[\text { TONY }_{\mathrm{CT}}[\text { invited SOPHIE } \mathrm{F}]\right]
$$

As in standard accounts of focus, I will assume that the alternatives of f-marked e-type expressions are simply sets of alternative expressions denoting individuals, here abbreviated as follows:

$$
\llbracket \text { Tony }_{\mathrm{CT}} \rrbracket^{f}=\llbracket \text { Sophie }_{\mathrm{F}} \rrbracket^{f}=\{x: x \in \text { HUMAN }\}
$$

Unlike standard accounts of focus, however, I will assume that the f-value of any non-f-marked expression is simply the same as its ordinary value. Instead of using pointwise function application (PFA) to compose each node in the f-value, f-marked expressions then compose with these expressions by first applying the following type-shifter to the f-marked expression. ${ }^{3}$

$$
\llbracket \odot_{S \alpha \rightarrow(\alpha \beta) \rightarrow S \beta} \rrbracket=\lambda m_{S \alpha} \cdot \lambda f_{(\alpha \beta)} \cdot \bigcup_{x \in m}\{f(x)\}
$$

This type-shifter allows us to compose sets (I will refer to these expressions as being of type $\mathrm{S} \alpha$ ) with ordinary expressions (i.e. expressions of type $(\alpha \beta)$ ). The result is again a set (i.e. an expression of type $\mathrm{S} \beta$ ). For instance, if we want to compose invite with Sophie $_{F}$, we first apply $\odot$ to the latter, and then compose the result with the verb:

3 Note that this operator is similar to the operator proposed in, e.g. Charlow (2019), in that it allows us to compose sets. It's different however, in that it combines $\mathrm{S} \alpha$ type expressions directly with $(\alpha \beta)$-type expressions. 
The role of focus marking in disjunctive questions

$$
\begin{aligned}
& \llbracket \odot \text { Sophie }_{F} \rrbracket^{f}=\lambda f . \bigcup_{x \in \text { HUMAN }}\{f(x)\} \\
& \llbracket \text { invited }\left[\odot \operatorname{Sophie}_{F}\right] \rrbracket^{f}=\bigcup_{x \in \text { HUMAN }}\{\lambda y . \mid y \text { invited } x \mid\}
\end{aligned}
$$

In this way, we can "percolate" alternatives up the tree without having to make use of PFA. This is helpful, because it allows us to combine multiple sources of alternatives-as we see in CT-F sentences. Successive application of $\odot$ will then ensure that, in these cases, the result is a nested set of alternatives. This would be impossible in a standard account of focus marking that only relies on PFA, because PFA would never give us an expression of a higher type if we combine two expressions that are sets themselves.

To see how this works out, we only need to solve one wrinkle which is that, as is, we're not able to compose sets with sets, so we first have to turn the set of type $S($ et $)$ corresponding to the VP in this case into a function of type eSt. We do this using the following operator:

$$
\llbracket \oplus_{S(\alpha \beta) \rightarrow \alpha S \beta} \rrbracket=\lambda f_{S(\alpha \beta)} \cdot \lambda x_{\alpha} \cdot \bigcup_{g \in f}\{g(x)\}
$$

Now we can apply $\llbracket \odot\left[\right.$ Tony $\left._{C T}\right] \rrbracket$ to the output of this, and we end up with a nested set of alternatives, exactly as we expect.

$$
\llbracket \odot\left[\text { Tony }_{C T}\right] \oplus\left[\operatorname{invited}\left[\odot \text { Sophie }_{F}\right]\right] \rrbracket^{f}=\bigcup_{y \in \text { HUMAN }}\left\{\bigcup_{x \in \text { HUMAN }}\{\mid y \text { invited } x \mid\}\right\}
$$

In this way, we can thus easily obtain the intuitive effect of contrastive topics, without having to stipulate a new type of PFA, a new type of movement (as in Constant (2014)), or having to assume that contrastive topics give rise to flatter f-values which are simply sets of propositions (as in Wagner (2012)).

To generalize this account to contrastive topics in questions like (24a), we only need to do one thing, which is to assume that the wh-elements have an f-value that is identical to those of the focus marked names we already saw above. I will assume that wh-items introduce alternatives in both their f-value and o-value, while f-marked expressions only introduce alternatives in their f-value. Concretely, I assume the following entry for who:

$$
\llbracket \mathrm{who} \rrbracket^{o}=\llbracket \mathrm{who} \rrbracket^{f}=\{x: x \in \text { HUMAN }\}
$$

Although I do not claim that wh-items are f-marked themselves, I will assume here that because they introduce alternatives in their f-value, they participate in the same scopal configuration with contrastive topics as foci do. Therefore, in order for the contrastive topic to be interpreted correctly, I'm assuming the following LF for (24a).

$$
\text { [TONY } \left._{\mathrm{CT}}\right]_{i} \text { Who did } x_{i} \text { invite? }
$$

Since wh-elements like who thus have the exact same effect as f-marked e-type expressions, we end up with the exact same focus value as for the CT-F answer above. This means that, when asking a CT-marked wh-question, the speaker is presupposing a QUD which is the same as the QUD presupposed by the answer. In other words, asking such a question signals that this question is part of a bigger strategy that the addressee would like to see resolved: question $\mathrm{Q}$ with contrastive topic marking not only raises the issue $\llbracket Q \rrbracket^{o}$, but also presupposes the presence of a QUD $\subseteq \llbracket Q \rrbracket^{f}$. In the next section I will show how we can use the same 
Hoeks

mechanism to derive the contribution of CT-marking in PolQs too.

\subsection{CT-alternatives in PolQs}

Like I did for contrastive topics in WhQs, I will first derive the f-value of CT-marked PolQ answers, and then apply the same mechanisms to CT-marked PolQs themselves. Recall the relevant question/answer pair, repeated here in (36).

$$
\begin{aligned}
& \text { a. A: Did SoPHIE } \\
& \text { b. B: } \text { SOPHIE }_{\mathrm{CT}}^{\mathrm{L}+\mathrm{H}^{*}}{ }^{\mathrm{L}} \text { DID }_{\mathrm{F}}^{\mathrm{H}^{*}} \text {, but TONY } \mathrm{TONT}_{\mathrm{CT}}^{\mathrm{L}+\mathrm{H}^{*}} \text { DIDN }^{\prime} \mathrm{T}_{\mathrm{F}}^{\mathrm{H}}{ }^{*} \mathrm{~L}-\mathrm{L} \%
\end{aligned}
$$

Adopting the assumptions we made above the for CT-marking in WhQ, I'm assuming the following LF for CT-marked answers to PolQs:

$$
\sim\left[\text { SOPHIE }_{\mathrm{CT}}\left[\operatorname{DID}_{\mathrm{F}}\langle\text { go to the party }\rangle\right]\right.
$$

The derivation for (37) will proceed in the exact same way as we saw for WhQs. I will make the straightforward assumption here that the focus marking on did is a verum focus triggering a positive and a negative alternative, in this particular case denoting the following set:

$$
\llbracket \operatorname{did}_{\mathrm{F}} \rrbracket^{f}=\{\lambda p \lambda y \cdot|p(y)|, \lambda p \cdot \lambda y \cdot|\neg p(y)|\}
$$

In order for this verum focus to compose with the ellided verb phrase, we again first apply $\oplus$, yielding (39) and, then after composing with (40) we end up with (41).

$$
\begin{aligned}
& \llbracket \oplus \operatorname{did}_{\mathrm{F}} \rrbracket^{f}=\lambda p \cdot\{\lambda y \cdot|p(y)|, \lambda y \cdot|\neg p(y)|\} \\
& \llbracket \text { go to the party } \rrbracket=\lambda y \cdot \mid y \text { go to the party } \mid \\
& \llbracket\left[\oplus \operatorname{did}_{\mathrm{F}}\right]\langle\text { go to the party }\rangle \rrbracket^{f}=\{\lambda y \cdot \mid y \text { go to the party }|, \lambda y \cdot| \neg y \text { go to the party } \mid\}
\end{aligned}
$$

To then apply this to the CT-marked subject, we again first apply $\oplus$ to the verum focused VP, and $\odot$ to the subject itself, thus giving us:

$$
\begin{aligned}
& \left.\llbracket \odot\left[\text { Sophie }_{\mathrm{CT}}\right] \oplus\left[\left[\oplus \operatorname{did}_{\mathrm{F}}\right]\langle\text { go to the party }\rangle\right]\right]^{f} \\
& =\bigcup_{y \in \text { HUMAN }}\{\{\mid y \text { go to the party }|,| \neg y \text { go to the party } \mid\}\}
\end{aligned}
$$

In other words, we will end up with a set of polar questions of the form Did x bring something? Just like the CT-F answer in (24b) gave us a set of wh-questions, a CT-F answer with verum focus will give us a set of polar questions. This makes sense intuitively, because this corresponds to a setting in which the speaker is addressing the superquestion that asks, for every individual, whether or not they went to the party. The answer in (36b) itself then resolves two such questions, by stating that Tony didn't go, but Sophie did.

Just as we saw for CT-marked wh-questions, the derivation of CT-marked PolQs will be exactly the same as the derivation for their corresponding answers. That is, analogous to the proposed analysis of wh-items, I will assume that the the question operator $Q$, which is responsible for turning a sentence into a polar question, also introduces alternatives in both its o-value and f-value. 
The role of focus marking in disjunctive questions

$$
\llbracket Q \rrbracket^{o}=\llbracket Q \rrbracket^{f}=\llbracket \operatorname{did}_{\mathrm{F}} \rrbracket^{f}=\{\lambda p \lambda y \cdot|p(y)|, \lambda p \cdot \lambda y \cdot|\neg p(y)|\}
$$

Note that because of this, the effect of the question operator in the f-value, will be exactly the same as we saw above for the verum focus on $\mathrm{did}$.

I will then assume the LF for CT-marked questions in (44). Note that, in order for the contrastive topic reading to arise, we need to assume again that the contrastive topic itself scopes over the other expression introducing alternatives, in this case the $Q$ operator.

$$
\sim\left[\text { SOPHIE }_{\mathrm{CT}}\right]_{i} \mathrm{Q}\left[\operatorname{Did} \mathrm{x}_{i}\right. \text { go to the party ] ] }
$$

The above LF will then yield the exact same f-value as we saw for its corresponding CT-F marked answer, thus signalling that the PolQ itself belongs to a larger QUD which asks of each salient individual, whether or not they went to the party.

\subsection{Focus marking in PolQs}

The CT-marked PolQ discussed in the previous section gave rise to a QUD which was a set of PolQs, but in this section I will argue that PolQs can contain simple foci too. That is, I will argue that the PolQ in (45) is more naturally analyzed as a focus, not a contrastive topic.

$$
\text { Did SOPHIE } \mathrm{H}^{\mathrm{H}} \text { win the race } \mathrm{H}^{\mathrm{H}}
$$

In assertions, we can easily distinguish between a focus and a contrastive topic because both typically have different prosodic properties. However, since PolQs like (45) generally come with a rising boundary tone independently of whether or not they contain a focus, it is more difficult to distinguish between foci and CTs in these questions (Kamali \& Krifka 2020).

However, since we've already established above that question/answer pairs become infelicitous when the answer presupposes a different QUD as the question, we can instead use the prosody of the answer to give us a clue about the underlying focus structure of the question itself. That is, note that (46a) and (46b) would form a natural question/answer pair, but note that, providing an answer that instead involves both CT and F-marking as in (46c), would not.

$$
\begin{aligned}
& \text { a. A: Did SOPHIE } \mathrm{H}^{\mathrm{H}} \text { win the race }{ }^{\mathrm{H} \%} \\
& \text { b. B: No, TONY } \mathrm{H}_{\mathrm{F}}^{\mathrm{H}^{*}} \text { did. } \\
& \text { c. B: \#No, but TONY } \mathrm{CT}_{\mathrm{CT}}^{\mathrm{L}+\mathrm{H}^{*}} \operatorname{DID}_{\mathrm{F}}^{\mathrm{H}{ }^{*} \%} \text {. }
\end{aligned}
$$

In contrast, the question/answer pair in (47a) and (47c) seems felicitous, but (47a) and (47b) does not.

$$
\begin{aligned}
& \text { a. A: Has SOPHIE } \mathrm{CT}_{\mathrm{CT}}^{\mathrm{L}+\mathrm{H}^{*}} \text { voted already }{ }^{\mathrm{H} \%} \\
& \text { b. B: \#No, TONY } \mathrm{H}_{\mathrm{F}}^{*} \text { did. } \\
& \text { c. B: No, but TONY }{ }_{\mathrm{CT}}^{\mathrm{L}+\mathrm{H}^{*}} \operatorname{DID}_{\mathrm{F}}^{\mathrm{L} \%}
\end{aligned}
$$

Intuitively, the answer in (47b) is infelicitous as a response to (47a), because it seems to come with the wrong presuppositions: It seems to presuppose that there was one person who has voted, and therefore that speaker A wants to know who that person was. This seems like a 
rather odd QUD to entertain, because there may be multiple people who have voted, or perhaps even no one at all. In this case, assuming that the QUD takes the form of a set of PolQs makes more sense, because such a QUD assumes that the answer to each PolQ is independent.

This, I take, will be the crucial difference between focus and contrastive topic marking in PolQs: While contrastive topic marking gives rise to an independence presupposition, focus marking gives rise to an existential presupposition. That is, simply by virtue of assuming a QUD which is a set of PolQs, the speaker presupposes that the answer to each PolQ is independent from answers to other PolQs. More concretely, by raising such a QUD, the speaker suggests that a scenario in which each PolQ is answered with "no" would be a possible resolution of the QUD, and so would a scenario in which every PolQ is answered with "yes". Thus, QUDs arising from CT-marking do not jointly presuppose that one of the f-alternatives is true.

Crucially, an independence presupposition is less natural for (46a), because usually in races there is exactly one winner. The answer in (46c) therefore forms an odd response to this question, because it presupposes a QUD which is a set of PolQs.

In short, these examples show that although, prosodically, foci and contrastive topics might be hard to tell apart, specific contexts may rule out a focus or a contrastive topic interpretation, because each of these types of f-marking give rise to different presuppositions. ${ }^{4}$

So how do we derive this difference? As already stated above, foci are those expressions that either occur under the scope of a contrastive topic, or occur on their own. If we analyze (47) as obligatorily being in instance of the latter case we would therefore take this question to have the following LF:

$$
\mathrm{Q} \text { Did } \sim\left[\mathrm{SOPHIE}_{\mathrm{F}} \text { win the race }\right] \text { ? }
$$

In this case, the question operator has to fall outside the scope of $\sim$, meaning that the alternatives generated by the focus on Sophie will not interact with the polar question alternatives coming from $Q$. The $\mathrm{f}$-value of the prejacent of $\sim$ will therefore end up being the following set of alternatives:

$$
\llbracket \text { SOPHIE }_{\mathrm{F}} \text { win the race } \rrbracket^{f}=\{\mid x \text { win the race } \mid: x \in \text { HUMAN }\}
$$

Thus, the presupposed QUD corresponding to this polar question will itself be a WhQ of the form Who won the race? Along with many accounts of focus, I argue that the presence of this WhQ as the presupposed QUD gives us an existential presupposition for free. Since the alternatives in this WhQ are the only ones signalled to be relevant to the speaker, the speaker already presupposes that there is an individual who won the race, and they want to know who that individual is. To further bring out the contrast between contrastive topics and regular foci, I will moreover make the standard assumption that focus marking comes with an additional exhaustive presupposition which contrastive topics lack (Hara \& van Rooij 2007; Tomioka 2010a,b; Wagner 2012; Constant 2014) . That is, when an utterance contains only F-marking, I

4 Independently, a similar claim is made by Kamali \& Krifka (2020) to account for PolQs in Turkish, in which the difference between focus and contrastive topic-marked PolQs becomes clear in the position in which the question particle $-m I$ shows up. 
will assume that each focus alternative will be exhaustified. For now, it will suffice to assume a simple exhaustification operator as in (50), which makes use of the prune operator as proposed by Menéndez-Benito (2005).

$$
\begin{array}{ll}
\text { a. } & \llbracket \operatorname{exh} \varphi \rrbracket^{f}=\left\{\operatorname{prune}\left(\alpha, \llbracket \varphi \rrbracket^{f}\right): \alpha \in \llbracket \varphi \rrbracket^{f}\right\} \\
\text { b. } & \operatorname{prune}(\alpha, \mathrm{A})=\{w: w \in \alpha \text { and } w \notin \beta \text { for any } \beta \in A \text { such that } \alpha \nsubseteq \beta\}
\end{array}
$$

That is, when applied to a set of f-alternatives $\llbracket \varphi \rrbracket^{f}$, exh "prunes" every alternative $\alpha$ in $\llbracket \varphi \rrbracket^{f}$ such that it no longer overlaps with any other alternative in $\llbracket \varphi \rrbracket^{f}$ not already containing $\alpha$.

Summing up, the difference between (46) and (47) is that the first will presuppose a QUD which is a WhQ, while the second will presuppose a QUD which is a set of PolQs. The reason for this difference in the shape of the QUD is that each of these come with their own presuppositions: A WhQ comes with both an exhaustive and an existential presupposition, but a set of PolQs comes with neither. Context, and the specific form of the question itself may therefore indicate whether we are dealing with focus or contrastive topic marking: such an existential presupposition would not make sense in (47) and an independence presupposition would not make sense in (46). Because of these differences in presupposed QUD, possible answers are also affected: We need answers to presuppose the same QUD as the questions, otherwise the question/answer pair will be incoherent.

In the next section we will see that just like PolQs, OpenQs are ambiguous between a focus and a contrastive topic reading. In contrast, we'll see that ClosedQs are not ambiguous in this way, and only allow for an interpretation in which the two pitch accents are a result of focus marking. For both types of AltQs, our focus-sensitive version of proper answerhood will then take care of the fact that these questions allow for different types of answers.

\section{Back to disjunctive questions}

Finally, we return to our main pattern of interest - that of disjunctive questions. Before moving on to the derivation of OpenQs and ClosedQs, I will first discuss disjunctive PolQs, which will be analyzed exactly in the same way as simple PolQs but, unsurprisingly, they will always contain a disjunction. These disjunctive PolQs will never have focus marking on the disjuncts, but they allow for focus or contrastive topic marking elsewhere. In contrast, OpenQs and ClosedQs will always involve some form of f-marking on the disjuncts.

\subsection{Disjunctive PolQs: lack of focus marking}

In their simplest form, disjunctive PolQs have the following LF.

Q [ Did Tony drink tea or coffee ]

The entry I will be using for disjunction will be one that when applied to non-focused disjunctions, will give us a maximally boring generalized disjunction interpretation. To make sure disjunction can also be applied to f-marked disjuncts in the case of OpenQs and ClosedQs, I assume that it corresponds to union when applied to expressions that are sets of conjoinable types. I thus define disjunction as follows: 
(52) Generalized disjunction for any $\alpha, \beta$ of conjoinable type $\tau, \llbracket \alpha$ or $\beta \rrbracket=\llbracket \alpha \rrbracket \sqcup \llbracket \beta \rrbracket^{5}$

a. For $T_{1}, T_{2} \in D_{t}, T_{1} \sqcup T_{2}=T_{1} \vee T_{2}$

b. For $f_{1}, f_{2} \in D_{(\sigma \tau)}, f_{1} \sqcup f_{2}=\lambda s_{\sigma} \cdot f_{1}(s) \vee f_{2}(s)$

c. For $f_{1}, f_{2} \in D_{S \tau}, f_{1} \sqcup f_{2}=f_{1} \cup f_{2}$

Given this entry for disjunction, the interpretation of (51) will thus be what we would expect:

$$
\llbracket(51) \rrbracket^{o}=\{\mid \text { Tony drank tea or coffee }|,| \neg \text { Tony drank tea or coffee } \mid\}
$$

Of course, a PolQ reading also arises when we have we have focus or contrastive topic marking outside of the disjunction, as in (54):

Did TONY F/CT $_{\text {drink tea or coffee? }}$

The derivation for these is analogous to those of simple PolQs, and will thus result in a presupposed QUD which is either a set of PolQs as in (55a) or a WhQ as in (55b).

a. $Q U D \subseteq\{\{\mid x$ drank tea or coffee $|,| \neg x$ drank tea or coffee $\mid\}: x \in$ HUMAN $\}$

b. $Q U D \subseteq\{\mid$ (only) $x$ drank tea or coffee $\mid: x \in$ HUMAN $\}$

In the next section, we will see that we can now account for OpenQs and ClosedQs as well.

\subsection{Foci and contrastive topics in OpenQs}

\subsubsection{F-marking in OpenQs}

The above will now allow us to analyze OpenQs like (4), here repeated as (56) as well.
a. A: Did Tony drink $\operatorname{COFFEE}_{\mathrm{F}}^{\mathrm{H}^{*}}$ or $\mathrm{TEA}_{\mathrm{F}}^{\mathrm{H}^{*}}$ yesterday morning? ${ }^{\mathrm{H} \%}$
b. B: No, he drank WATER F $_{\mathrm{F}}$
c. B: Yes, he drank COFFEE/TEAF

Assuming that the accents here reflect F-marking, the question in (4) will have the LF in (57).

$$
\mathrm{Q}\left[\sim \operatorname{exh}\left[\operatorname{Did} \text { Tony drink TEA } F \text { or } \operatorname{COFFEE}_{F}\right]\right]
$$

Just as we saw for PolQs, the QUD presupposed here will correspond to a WhQ. To derive this, we only need to put together the pieces that have already been introduced above. In both (57) the disjunction as a whole will denote the following set of individuals in its f-value:

$$
\llbracket \text { DisjP } \rrbracket^{f}=\{x: x \in \text { DRINKS }\}
$$

In other words, since disjunction is defined as union for sets, the f-value of the whole disjunction will be identical to the $\mathrm{f}$-value of each of the disjuncts. Therefore, the rest of the derivations for (58) will be exactly the same as those for the corresponding PolQ with F-marking. Again,

5 Where $t$ is a conjoinable type and if $\tau$ is a conjoinable type $(\sigma \tau)$ is a conjoinable type for any $\sigma$, and if $\tau$ is a conjoinable type $S \tau$ is a conjoinable type 
The role of focus marking in disjunctive questions

we will thus end up with the following QUD.

$$
Q U D \subseteq\{\mid \text { Tony drank (only) } x \mid: x \in \text { DRINKS }\}
$$

Note, however, that the ordinary value of an OpenQ like (56) will simply be as in (60).

$$
\llbracket(56) \rrbracket^{o}=\{\mid \text { Tony drank tea or coffee }|,| \neg \text { Tony drank tea or coffee } \mid\}
$$

As expected, focus marking will not have any effect on the ordinary value of this question. The only expression that therefore introduces alternatives is the question operator Q, thus yielding a set containing one positive and one negative alternative. If we would therefore only take the ordinary value of this question into account, we would predict that this question has the same answerhood conditions as the corresponding polar disjunctive question. However, recall that our focus-sensitive version of answerhood not only takes into account the ordinary value of the question, but also the QUD arising from its f-value. That is, focus-sensitive answerhood requires an answer $\llbracket A \rrbracket^{o}$ to resolve $\llbracket Q \rrbracket^{o}$ as well as $\llbracket Q \rrbracket^{f}$.

To thus answer both the PolQ in (60) as well as the QUD in (59), a negative answer stating that the disjunction is false or an affirmative answer stating that the disjunction is true will not suffice. Although such answers resolve the PolQ itself, they do not resolve the QUD. In contrast, any question that minimally resolves the WhQ will also resolve the PolQ itself. For instance, answering with Tony drank tea, resolves the PolQ as well as the QUD. Moreover, an answer like No, Tony drank water, will also be felicitous, because it states that the disjunction is false, and at the same time it answers the WhQ corresponding to the QUD.

We thus derive the correct answerhood conditions, but we also predict that any answer to such an OpenQ needs to involve F-marking to make it congruent to the QUD. Moreover, by relying on focus marking to capture these patterns, we also easily account for the both the existential and exhaustivity presuppositions that these questions bring along.

\subsubsection{CT-marking in OpenQs}

If one would interpret the pitch accents in an OpenQ instead as contrastive topics, we make slightly different predictions in terms of its possible answers. To see this, consider the example in (61a). Now, the LF of an OpenQ like this, would be as follows, where again, the contrastive topic marking on each of the disjuncts ensures that these take scope over the question operator.

$$
\begin{aligned}
& \text { a. Has TONY } \mathrm{CT}^{\mathrm{L}+\mathrm{H}^{*}} \text {, or SOPHIE } \mathrm{CT}^{\mathrm{L}+\mathrm{H}^{*}} \text { voted already }{ }^{\mathrm{H} \%} \\
& \text { b. } \sim \text { [TONY }_{\mathrm{CT}} \text { or SOPHIE } \mathrm{CT}_{i}\left[\mathrm { Q } \left[\text { has } \mathrm{x}_{i}\right.\right. \text { voted already ] ] }
\end{aligned}
$$

As in CT-marked PolQs, we therefore end up with the following presupposed QUD, which again will correspond to a set of PolQs.

$$
Q U D \subseteq\{\{\mid x \text { has voted }|,| \neg x \text { has voted } \mid\}: x \in \text { HUMAN }\}
$$

In terms of possible answers then, note that again, a simple affirmative answer will not resolve any of the PolQs within the superquestion presupposed as the QUD. Similar to the parse with focus marking on the disjuncts, a contrastive topic parse will also predict that answers 
corresponding to each of the disjuncts are fine, because each of these will provide positive answers to at least one polar question within the QUD. Hence, we predict that both (63b) and (63c) are felicitous responses to a question like (63a).
a. A: Has TONY ${ }_{\mathrm{CT}}$, or SOPHIE $\mathrm{CT}_{\mathrm{Ct}}$ voted already?
b. B: Yes, TONY ${ }_{\mathrm{CT}}$ DID $_{\mathrm{F}}$.
c. B: TONY ${ }_{\text {CT }}$ DID $_{F}$, but SOPHIE CT $_{\text {DIDN' }} T_{F}$
d. B: No, NEITHER OF THEM CT $_{\text {DID }}$

However, note that we predict that a negative response which states that the disjunctive statement is false as in (63d) will also resolve both the question itself and the QUD. This is because it provides negative answers to two polar questions within the superquestion that is presupposed, and will therefore satisfy the condition of resolving at least one subquestion. That is, it resolves both the PolQ Did Tony finish the assignment? and the PolQ Did Sophie finish the assignment? by providing a negative response to both.

\subsection{Foci in ClosedQs}

ClosedQs as in (3), repeated here in (64) are similar to OpenQs in that they involve pitch accents on each disjunct. Such ClosedQs are crucially different from OpenQs in that they obligatorily involve a final pitch accent which is falling, followed by a falling boundary tone.

$$
\begin{aligned}
& \text { a. A: Did Tony drink COFFEE }{ }^{L+H *} \text { or } \text { TEA }^{H+L *} \text { yesterday morning? }{ }^{L-L \%} \\
& \text { b. B: He drank COFFEE/TEA }
\end{aligned}
$$

The account of ClosedQs will therefore be very similar to that of OpenQs except that I add one more component to the above, namely the assumption that a final falling pitch accent has the effect of domain restriction. I assume that the falling pitch accent results in a restricted set of focus alternatives which only consists of those alternatives that are explicitly mentioned (see Meertens, Eggers \& Romero (2019) for a proposal along similar lines). This idea is inspired by accounts of falling intonation in disjunctions like those proposed in Zimmermann (2000), or Biezma (2009), in which disjunctive statements are assumed to correspond to lists that can either be "open" or "closed off". In the latter case, it is indicated that there are no relevant unmentioned list items which have the property in question.

I thus implement this intuition here with respect to focus alternatives: I will assume that the falling intonation signals that there are no focus alternatives other than those explicitly mentioned which are relevant in the specific context. Even though list closure is often tied to the falling boundary tone Biezma (2009), I will suggest here that it is actually the final pitch accent which crucially signals the presence of a closure operator $\Gamma$, that scopes over the disjunction and restricts the domain f-alternatives to those entailing its o-value.

$$
\begin{array}{ll}
\text { a. } & \llbracket \Gamma \varphi \rrbracket^{f}=\left\{\alpha \in \llbracket \varphi \rrbracket^{f}: \alpha \subseteq \llbracket \varphi \rrbracket^{o}\right\} \\
\text { b. } & \llbracket \Gamma \varphi \rrbracket^{o}=\llbracket \varphi \rrbracket^{o}
\end{array}
$$

If $\varphi_{\mathrm{F} 1}, \ldots, \varphi_{\mathrm{FN}}$ are list items, $\Gamma$ is spelled out prosodically as an $\mathrm{H}+\mathrm{L}^{*}$ accent on $\varphi_{\mathrm{FN}}$, and a 
falling boundary tone. ClosedQs like (64), therefore have the following structure:

$$
Q\left[\sim \operatorname{exh}\left[\text { Did Tony drink [ [ COFFEE } \text { or TEA }_{\mathrm{F}}\right] \Gamma\right] \text { yesterday morning ] ] }
$$

The f-value of the closed disjunction will thus be as in (67b), while it's o-value will simply be the standard disjunctive meaning as in (67a).

$$
\begin{array}{ll}
\text { a. } & \llbracket \text { DisjP } \rrbracket^{o}=\lambda p \cdot \mid p(\text { tea }) \vee p(\text { coffee }) \mid \\
\text { b. } & \llbracket \text { Dis } j P \rrbracket^{f}=\{x: x \in\{\text { tea, coffee }\}
\end{array}
$$

The disjunctive alternatives again percolate up the tree, and will then give rise to the QUD in (68b). Note that the ordinary value of (64) will again simply be the polar question in (68a).

$$
\begin{array}{ll}
\text { a. } & \llbracket(64) \rrbracket^{o}=\{\mid \text { Tony drank tea or coffee }|,| \text { Tony drank neither } \mid\} \\
\text { b. } & Q U D \subseteq\{\mid \text { Tony drank tea (and no coffee) }|,| \text { Tony drank coffee (and no tea) } \mid\}
\end{array}
$$

As we saw in f-marked OpenQs, this presupposed QUD will affect what will count as a proper answer. Recall that proper answerhood required that an answer to any f-marked question resolves not only the question itself, but also its presupposed QUD. Concretely, this means that answering with the disjunctive statement Tony drank tea or coffee will not suffice in this case because it doesn't resolve the QUD. Therefore, an answer to (64) will have to be either entail Tony drank tea or Tony drank coffee. Answering with an proposition that does not entail any of the disjuncts will not do because such answers will not address the QUD. This also means that an answer like Tony drank water, will not answer the QUD either.

Due to $\Gamma$, we also derive that ClosedQs like (64) automatically come with a presupposition that either of the two disjuncts is true. Moreover, assuming that the interpretation in ClosedQs comes from the fact that they are F-marked, also allows us to capture the intuition that these questions are typically interpreted exhaustively. Together, we thus capture the intuition that ClosedQs come with the presupposition that exactly one of the disjuncts is true.

\section{Conclusion}

In sum, this paper provides an account of the effect of prosody on the interpretation of disjunctive questions that solely relies on effects of focus marking. Differences in the prosodic realization of these questions give rise to different QUDs, which can in turn affect what constitutes a possible answer. To get there, I first had to recast question/answer congruence into a requirement on answers to be congruent to the contextually salient QUD introduced by f-marked questions. This meant that answers to such questions need to address this QUD, either by resolving at least one subquestion within the QUD, or by resolving that QUD itself.

The main claim that was put forward was therefore that the differences in interpretation between PolQs, OpenQs and ClosedQs do not come from the semantics proper, but can instead be derived via general conditions on focus congruence. Along the way, I introduced a method for computing f-values that allows for the generation of CT and F-alternatives. Using this approach, we can account for the interpretation of disjunctive questions, but more importantly, we can also understand how they fit into a larger paradigm of focus-marked questions. 
Hoeks

\section{References}

Bartels, Christine. 1999. The Intonation of English Statements and Questions: A Compositional Interpretation. New York: Routledge.

Beck, S. 2006. Intervention effects follow from focus interpretation. Natural Language Semantics 14(1). 1-56.

Biezma, Maria. 2009. Alternative vs. polar questions: the cornering effect. In Satoshi Ito \& Ed Cormany (eds.), Semantics and Linguistic Theory (SALT) 14, CLC Publications.

Büring, Daniel. 2003. On D-trees, Beans, and Accents. Linguistics and Philosophy 26. 511-545.

Büring, Daniel. 2016. Intonation and meaning. Oxford University Press.

Charlow, Simon. 2019. The scope of alternatives: indefiniteness and islands. Linguistics and Philosophy doi:10.1007/s10988-019-09278-3.

Ciardelli, Ivano, Jeroen Groenendijk \& Floris Roelofsen. 2018. Inquisitive Semantics. Oxford University Press.

Constant, Noah. 2014. Contrastive topic: Meanings and realizations: University of Massachusetts $\mathrm{PhD}$ dissertation.

Han, Chung-hye \& Maribel Romero. 2004. Disjunction, focus, and scope. Linguistic Inquiry 35(2). 179-217.

Hara, Yurie \& Robert van Rooij. 2007. Contrastive topics revisited: A simpler set of topicalternatives. NELS 38.

Hoeks, Morwenna. 2019. Focus alternatives in alternative questions. In European Summer School for Logic, Language and Information (ESSLLI) 2019 Student Session, 1-12.

Jackendoff, Ray. 1972. Semantic Interpretation in Generative Grammar. MIT Press.

Kamali, Beste \& Manfred Krifka. 2020. Focus and contrastive topic in questions and answers, with particular reference to Turkish. Theoretical Linguistics 46(1-2). 1-71.

Meertens, Erlinde. 2019. Prosody in disjunctive questions: Introducing class. University of Pennsylvania Working Papers in Linguistics 25(1).

Meertens, Erlinde, Sophie Eggers \& Maribel Romero. 2019. The role of multiple accent in alternative questions. In M. Teresa Espinal (ed.), Sinn und Bedeutung 23, 179-196.

Menéndez-Benito, Paula. 2005. The grammar of choice. University of Massachusetts Amherst $\mathrm{PhD}$ dissertation.

Pruitt, Kathryn \& Floris Roelofsen. 2013. The interpretation of prosody in disjunctive questions. Linguistic Inquiry 44.632-650. doi:10.1162/LING_a_00141.

Roelofsen, Floris. 2015. The semantics of declarative and interrogative lists. Manuscript, ILLC, University of Amsterdam.

Roelofsen, Floris \& Sam van Gool. 2009. Disjunctive questions, intonation, and highlighting. Technical report, ILLC, University of Amsterdam.

Rooth, Mats. 1985. Association with focus. University of Massachusetts Amherst PhD dissertation.

Rooth, Mats. 1992. A theory of Focus interpretation. Natural Language Semantics 1. 75-116.

Tomioka, Satoshi. 2010a. Contrastive topics operate on speech acts. In C. Féry \& M. Zimmermann (eds.), Information structure: Theoretical, typological, and experimental per- 
The role of focus marking in disjunctive questions

spectives, University Press Oxford.

Tomioka, Satoshi. 2010b. A scope theory of contrastive topics. Iberia: An International Journal of Theoretical Linguistics 2(1). 113-130.

Wagner, Michael. 2012. Contrastive topics decomposed. Semantics and Pragmatics 5(8). $1-54$.

Zimmermann, Ede. 2000. Free choice disjunction and epistemic possibility. Natural Language Semantics 8(2). 255-290.

Morwenna Hoeks

Linguistics Department

University of California Santa Cruz

1156 High Street

Santa Cruz, CA 95064

USA

mhoeks@ucsc.edu 